\title{
Using motivational tactics to support children with reading disabilities
}

\author{
Hilary Scruton, John McNamara \\ Child and Youth Studies, Brock University, St. Catharines, Ontario, Canada
}

Email address:

hs10qw@brocku.ca (H. Scruton),jmcnamara@brocku.ca (J. McNamara)

\section{To cite this article:}

Hilary Scruton, John McNamara. Using Motivational Tactics to Support Children with Reading Disabilities. International Journal of Elementary Education. Vol. 3, No. 4, 2014, pp. 92-97. doi: 10.11648/j.ijeedu.20140304.11

\begin{abstract}
This paper presents Reading Rocks, a literacy program for children with reading disabilities. In addition to component literacy skill areas (i.e. phonics, sight word vocabulary) Reading Rocks focuses on motivational tactics aimed to promote children's task understanding, goal setting, and monitoring. The motivational tactics adopted by the Reading Rocks program correspond with the self-regulated learning model and are applied to reading interventions. This paper reviews the theoretical underpinnings of self-regulated learning and reading disabilities and also presents illustrations and descriptions of how motivational tactics within Reading Rocks were designed.
\end{abstract}

Keywords: Learning Disabilities, Motivation, Literacy

\section{Introduction}

Although the majority of children develop reading skills without difficulty, approximately $20 \%$ of children experience significant challenges with the reading process (Snow, Burns, \& Griffin, 1998). Reading difficulties can be attributed to a number of factors including environmental disadvantage, second language learners, and/or cognitive processing impairments commensurate with diagnoses such as learning disabilities (Case, Speece, Molloy, 2003; Crosnoe, Leventhal, Wirth, Pierce, \& Pianta, 2010; Morris, Lovett, Wolf, Sevcik, Steinbach, Frijters, \& Shapiro, 2012). Learning disabilities, and particularly reading disabilities have received a great deal of attention over the past decades as stakeholders have realized the negative effect reading disabilities can have on not only academic achievement, but also secondary characteristics such as depression, anxiety, and a host of other mental health related illnesses. This paper presents an intervention approach for children with reading disabilities that considers not only the academic challenges faced by vulnerable readers but also the motivational affects that reading difficulties can present.

\section{Reading Difficulties and Motivation}

Research has demonstrated that overtime the pervasive cumulative effects of the lived-experience of having a reading disability can have a detrimental effect on the well-being of children and youth. A review of the research in this area highlights findings indicating that higher scores on inventories measuring depression have been found in children with reading disabilities as young as 8 years of age and continuing into adolescence (Wilson, Armstrong, Furrie \& Walcot, 2009). The National Longitudinal Study of Adolescence Health reported that $5.7 \%$ of adolescence with school-identified reading disabilities had attempted suicide. In addition, as individuals with reading disabilities move into adulthood, it has been found that $25 \%$ of the population in the federal prison system has been identified as having reading disabilities (Ostiguy, 2000). In general, research has elucidated that children and youth with reading disabilities are at increased risk for mental health disorders including stress, anxiety, depression, and suicide. The chronic stress, prolonged anxiety, and depression associated with reading disabilities can lead to negative brain changes such as loss of synapses and retraction of dendrites that ultimately impact long term-cognitive functioning.

However, these negative developmental trajectories are not inevitable. It may be that mental health related issues can be avoided by early effective intervention focusing on academic skills, as well as issues of motivation and self-esteem. For the past several decades, researchers, practitioners, and concerned stakeholders have worked to establish effective interventions for children with reading disabilities (Snow et al. 1998). This 
work has culminated in firm understandings about the skill component areas where vulnerable readers struggle most significantly. Reports such as the National Reading Panel have suggested that the most effective approach to reading instruction for vulnerable readers is one that incorporated explicit instruction in phonemic decoding, systematic phonics instruction (i.e. letter sound understanding, phonics principles, etc.), vocabulary development, increasing reading fluency, and bolstering reading comprehension. Following the National Reading Panel's (2000) recommendations, most commercially published reading programs include instruction in these skill areas.

Holtzheuser, McNamara, and Short (2014) proposed that traditional literacy programs for vulnerable readers may be enhanced by incorporating self-regulated learning into reading intervention. This proposal was based on the notion that struggling readers may come to reading activities with perceptions that impact how they will engage in reading tasks. For instance, struggling readers may have encountered previously unsuccessful reading experiences that have led to internalizing a lower sense of self-efficacy and thus decreased motivation. Under these circumstances, it would be reasonable to expect that the same reader would approach subsequent reading tasks with perceptions that may mediate negative engagement (Holtzheuser et al., 2014). Thus, there is a need to utilize a self-regulated learning process to not only support the academic skills needed to read, but also to engage motivational processes that will support long-term reading success. Holtzheuser et al., (2014) suggest that the self-regulated learning model proposed by Winne and Hadwin (1998) is a useful tool in supporting the reading process for children with reading disabilities. Specifically, Winne and Hadwin (1998) describe a self-regulated learning process that engages learners' task understanding and perceived self-efficacy, goal setting, and monitoring and feedback.

The current paper forwards this work and introduces a reading intervention program that considers self-regulated learning within the context of a traditional reading program through the use of motivational tactics.

\section{Program Design}

\subsection{Reading Rocks and Motivational Tactics}

Reading Rocks is a literacy intervention approach aimed at supporting vulnerable readers designed by John McNamara, Ashley Short, and Hilary Scruton (2014) and offered widely throughout the Niagara Region of Ontario. Reading Rocks is best suited to be used as a one-on-one instructional approach however; it can be modified for small groups of readers at similar reading levels. The program is designed to be an 8-week intervention program that includes two, 1-hour sessions, weekly. Trained tutors delivering the program work individually with children who have been identified as having reading-based needs. The program is based on recommendations by the National Reading Panel (2000) suggesting that remedial literacy instruction be systematic and explicit, providing children with ample opportunity for individual feedback and practice with foundational literacy skills such as phonetic decoding, phonics, sight word vocabulary, fluency, and comprehension. Furthermore, the one-on-one instruction provided in the Reading Rocks program allows tutors to customize the program to best meet individual children's various needs.

The Reading Rocks program is designed to be delivered in a series of 1-hour tutoring sessions. Each hour session should be broken down into four 15-minute instructional blocks each corresponding with one literacy-based instructional component. For example, a 1-hour tutoring session would include a 15-minute block of phonics, followed by a 15-minute block of vocabulary, a 15-minute block of reading fluency, and ending with a 15-minute block of reading appreciation. The purpose of 15-minute block structure is twofold. First, research has demonstrated that short, intensive instructional sessions (10-15 minutes) are more effective than longer sessions (Vaughn \& Roberts, 2007). Second, the 15minute block structure in Reading Rocks is designed to be motivational. Within the program, children set out to meet instructional goals within set time periods. This process encourages children to engage with the task vigorously in order to meet their defined goal.

Reading Rocks is similar to traditional reading programs in that tutors focus on essential component skills required to teach children how to read; however, the program is unique as it also uses motivational tactics to engage children's self-regulated learning. Reading Rocks recognizes that within the spectrum of motivation there are several tactics that can be used to bolster children's motivation and increase their reading achievement. Specifically, within each instructional block (i.e. phonics, sight word instruction, etc.) Reading Rocks engages a number of motivational tactics that promote the self-regulated learning skills proposed by Winne and Hadwin (1998). The motivation tactics are engaged through the use of instructional workstations that tutors design and tailor to each child's needs.

\section{Motivational Tactics in Action}

\subsection{Instructional Workstations}

The instructional workstations used in Reading Rocks are the medium through which motivational tactics are explicitly illustrated for children. The instructional workstations are essentially graphic organizers where tutors and children collectively represent the achievement gained in the program. A unique aspect of the instructional workstation is that tutors and children build and develop workstations collectively as a team as the Reading Rocks program progresses. A completed instructional workstation as developed throughout the Reading Rocks program is illustrated in Figure 1. 


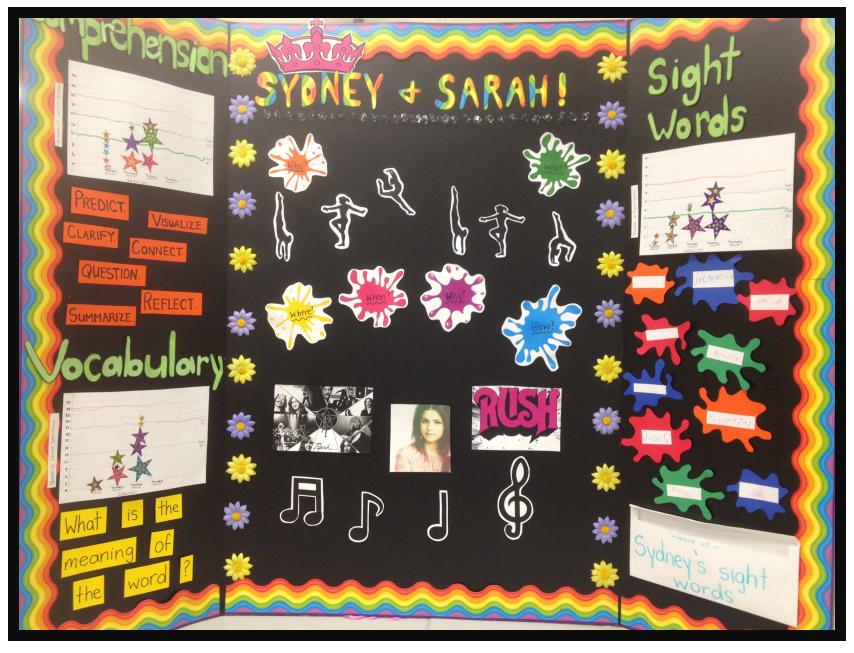

Figure 1. Completed Instructional Workstation.

\subsection{Task Understanding and Perceived Self-Efficacy}

As described by Holtzheuser et al. (2014) understanding the demands of a task is an important first step in the self-regulatory learning process as it sets the foundation for how a learner will engage. Reading Rocks instructional workstations engage children's perceptions of demands of the tasks and enable children's understanding about how their achievement and outcomes will be evaluated. The first step in the workstation design process is to build a basic instructional framework that illustrates the three skill component areas (i.e. phonics, sight words, and reading fluency) that the tutor and child with focus on throughout the duration of the program (Figure 2). By simply illustrating the basic frameworks of instruction and engaging children in a conversation about their perceptions around the skill areas, children begin the program developing a sense of ownership over their learning. In turn, the workstations begin to strengthen children's self-efficacy for their upcoming task prior to engaging with it (Holzheuser et al., 2014).

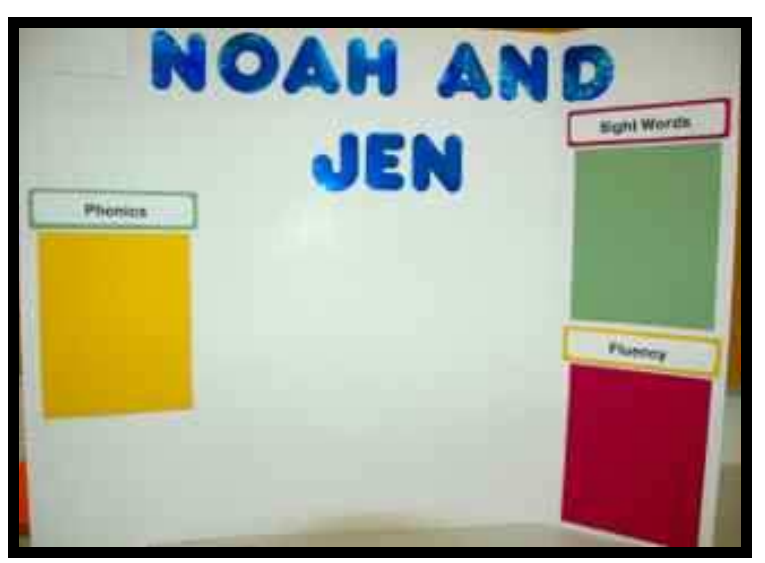

Figure 2. Example of Initial Skill Component Framework.

As tutors continue to become familiar with children, the instructional workstations become even more personalized thus creating a further sense of ownership. For example, in Figure 3 the tutor has designed a workstation based around the child's interest of soccer.



Figure 3. Example of Task Understanding with Workstation.

\subsection{Goal Setting}

Holtzheuser et al. (2014) describes goal setting as planning particular outcomes of learning or performances. Goal setting and attainment enhances motivation, performance during the task, and perceived self-efficacy (Bandura, 1997; Schunk, 2003). However, goal setting is often not a natural process that learners will engage in without instruction in how to set goals effectively. Setting unrealistic goals can lead to feelings of frustration and incompetence, resulting in a decrease of self-efficacy and self-esteem (Elliot et al., 2005). In contrast, when children consider a goal to be attainable, they are more likely to be committed in achieving that goal (Locke \& Latham, 2002). When children are provided with support and guidance in setting attainable goals they can reach and, in many cases, exceed their goals. Reaching and exceeding literacy goals gives children confidence in their reading abilities, increasing their self-efficacy and self-esteem, and ultimately motivate children to continue to engage in reading tasks. Within the Reading Rocks program, goal setting is utilized as an important tactic for motivating children to engage within their own learning in order to meet or exceed their goals. An important aspect of the goal setting process in Reading Rocks is that goals are collectively developed and set by both tutors and children. Goals are deliberately set to be challenging yet attainable allowing children to feel a sense of accomplishment when goals are met.

The goal setting process begins with tutors engaging children in a conversation about their current achievement levels and setting appropriate goals for future achievement. Specifically, at the beginning of each instructional block (i.e. phonics, sight words, etc.) tutors first take time to talk with children about their instructional goal for the session and also for a longer-term period (i.e. 2 or 3 sessions). Following this, tutors and children decide upon instructional goals. For instance, in Figure 5 the tutor and child set a goal of mastering 15 new sight words within three instructional sessions. In each case, tutors ensure that children reach their goal within the allotted time. To further motivate children, tutors reinforce accomplishments by providing feedback about the effort 
required to reach goals. In addition, tutors are encouraged to seek an external figure aside from themselves to recognize children's accomplishments, thus further creating a sense of self-efficacy and motivation. After this process, tutors and children set the next instructional goal accordingly as illustrated in Figure 4.



Figure 4. Goal Setting Process.

\subsection{Monitoring and Feedback}

An important aspect of self-regulatory and motivational processes is monitoring one's own progress and producing internal feedback about one's engagement with the task. Monitoring and providing feedback, either during the task or after the completion of the task, allows learners to reinterpret how they are engaging with the specific task and how the resulting outcomes of the task may inform their subsequent engagement with similar endeavors (Holtzheuser et al., 2014). In the context of reading, Zimmerman (2008) would suggest that strong readers often reflect on their reading task outcomes and compare their achievement with the task to the goals they set prior to engaging with the task. However, children with reading disabilities may experience several difficulties with the monitoring and feedback process. Due to past failures with reading-based tasks, children with reading disabilities may not engage in this process to the same extent as their typically achieving peers. This may be because struggling readers may not naturally engage strategically with their own learning and related to this, children with reading disabilities may lack the cognitive resources available for such strategic engagement (Margolis \& McCabe 2004). The instructional workstations in Reading Rocks address these potential challenges by providing children with external sourced monitoring and feedback. In other words, Reading Rocks tutors scaffold instruction in order to demonstrate how children can monitor their own learning and provide appropriate feedback. Subsequently, this feedback and monitoring process will increase children's self-efficacy and motivation.

Figure 5 illustrates how participating children are encouraged to point out their achievements on their workstation graph. In this case, this child is pointing out his reading fluency rate that has surpassed his instructional goal. In addition graphing, the workstation acts as a graphic organizer for any new sight words or phonics principles that are learned during the program. For instance, Figure 5 illustrates a number of sight words that the child has acquired. After achievements are graphed, tutors would engage children in a conversation about how their effort and effective use of instructional strategies led to their accomplishments. This conversation would be aimed at enabling children to understand that internal attributes such as effort and strategy use were primary contributing factors to his success thus creating an internal sense of efficacy and motivation.



Figure 5. Monitoring and Feedback.

A final aspect to the monitoring and feedback processes of Reading Rocks is transference of skills. Children participating in Reading Rocks take their instructional workstations home increasing the likelihood that the motivational tactics they acquired throughout the program will transfer to subsequent reading activities and also more general areas of academic achievement.

\section{Discussion and Conclusion}

Motivational tactics are important components of the learning process. Children with reading disabilities often experience significant challenges with their academic achievement and also their self-regulated learning. Specifically, children with reading disabilities tend to approach tasks with poor self-efficacy, do not engage in effective goal setting, and have negative internal monitoring and feedback systems. Reading Rocks, a literacy intervention program for vulnerable readers, combines the explicit teaching of effective reading-based skills (phonics, sight words, and fluency) with a focus on self-regulated learning through the use of instructional workstations and motivational tactics. Within the Reading Rocks program, tutors work with children to design and build an instructional workstation that acts as a graphic organizer of their self-regulated learning. As illustrated through this paper, tutors and children collaboratively develop a sense of task understanding, set challenging yet achievable goals, and track and monitor progress. Reading Rocks is aimed at engaging children's 
self-regulated learning processes and as such, increasing their motivation to engage in reading-based tasks - tasks that children with reading disabilities often find significantly challenging.

Future empirical research in this area will aim to measure the effects of motivational tactics such as those described in this paper. However, exploring the real-time use motivational tactics is challenging. Future research may consider collecting self-report data from children through think-aloud protocols such as those proposed by Bannert and Mengelkamp (2008). In general, think-aloud protocols have children talk-aloud while thinking, learning, and engaging in their literacy activities. Verbal traces are then analyzed for patterns and constructs. Although this approach has been criticized (see Afflerbach, 2000), it is considered by researchers to be a good method of collecting on-line processing associated with metacognitive learning.

The process of bridging a model of reading remediation and self-regulated learning holds important implications. First, research suggests that struggling readers often lack the self-efficacy to engage in the reading process. Following this, it is reasonable to expect that struggling readers are generally less motivated to engage in reading tasks. This is problematic in that vulnerable readers are learning alongside strong readers who are engaging with reading tasks. The differences in "willingness to engage" can create long-term gaps in achievement that are difficult to overcome. However, by improving struggling readers' self-efficacy by engaging their motivation through tactics such as those described in this paper, children may be more likely to engage in reading, and thus, progress through the reading process. A second implication is based on the data indicating that the gap between grade-level and struggling readers grows exponentially throughout elementary school years. By engaging vulnerable readers motivational processes, educators will be promoting at-risk readers' self-directed learning - an important process that will assist children in the long-term independent learning process. Finally, by facilitating the development of motivation, children can employ these skills in all areas of their education, to not only use reading as a tool for further learning, but to do so effectively and independently.

\section{Acknowledgements}

The authors would like to thanks the staff, facilitators, and volunteers at the Learning Disabilities Association of Niagara Region for their support with this project. Also, H. Scruton would like to acknowledge the financial support of the Social Sciences and Humanities Research Council of Canada.

\section{References}

[1] Afflerbach, P. (2000). Verbal reports and protocol analysis. In M. L. M. Kamil, P. B. Mosenthal, D. Pearson, \& R. Barr (Eds.), Handbook of reading research (Vol. III, pp. 163-179). Hillsdale, NJ: Erlbaum.
[2] Bandura, A. (1997). Self-efficacy: The exercise of control. New York: Freeman.

[3] Bannert, M \& Mengelkamp, C (2008). Assessment of metacognitive skills by means of instruction to think aloud and reflect when prompted. Does the verbalisation method affect learning? Metacognition Learning, 3, 39-58.

[4] Case, L. P., Speece, D. L., \& Molloy, D. E. (2003). The validity of a response-to-instruction paradigm to identify reading disabilities: A longitudinal analysis of individual differences and contextual factors. School Psychology Review, 32, $557-582$.

[5] Crosnoe, R., Leventhal, T., Wirth, R. J., Pierce, K. M., \& Pianta, R. C. (2010). Family socioeconomic status and consistent environmental stimulation in early childhood. Child Development, 81, 972-987.

[6] Elliot, A. J., Shell, M. M., Henry, K. B., \& Maier, M. A. (2005). Achievement Goals, Performance Contingencies, and Performance Attainment: An Experimental Test. Journal of Educational Psychology, 97(4), 630.

[7] Holtzheuser, S., McNamara, J., Short, A., (2014). Self-regulation and motivation in children at-risk for learning disabilities, Exceptionalities Education International, 24 (1), 2-17.

[8] Locke, E. A., \& Latham, G. P. (2002). Building a practically useful theory of goal setting and task motivation: A 35-year odyssey. American Psychologist, 57, 705-717.

[9] Margolis, H., McCabe, P. (2004) Self-efficacy: A key to improving the motivation of struggling learners. The Clearing House, 77, 6. 241-249.

[10] McNamara, J. Short, A. \& Scruton, H. (2014). Reading Rocks: An intervention program to support vulnerable readers, The Research Institute for Learning Differences, St.Cath., ON.

[11] Morris, R. D., Lovett, M. W., Wolf, M., Sevcik, R. A., Steinbach, K. A., Frijters, J. C., \& Shapiro, M. B. (2012). Multiple-Component Remediation for Developmental Reading Disabilities IQ, Socioeconomic Status, and Race as Factors in Remedial Outcome. Journal of Learning Disabilities, 45, 99-127.

[12] National Reading Panel (2000). Report of the national reading panel: Teaching students to read: An evidence-based assessment of the scientific research literature on reading and its implications for reading instruction: Reports of the subgroups. Bethesda, MD: National Institute of Child Health and Human Development, National Institutes of Health.

[13] Ostiguy, J. (2000). "The Cognitive Skills - Building and Reintegration Program", Let's Talk, vol. 25 no. 2. Sector Reports, Correctional Operations and Programs Sector, Correctional Service Canada.

[14] Schunk, D. (2003). Self-efficacy for reading and writing: Influence of modeling, goal setting, and self-evaluation. Reading and Writing Quarterly, 19, 159-172.

[15] Snow, C. E., Burns, M. S., \& Griffin, P. (1998). Preventing reading difficulties in young children. Washington, DC: National Academy Press.

[16] Vaughn, S., \& Roberts, G. (2007). Secondary interventions in reading. Teaching Exceptional Children, 39(5), 40-46. 
[17] Wilson, A. M., Armstrong, C. D., Furrie, A., \& Walcot, E. (2009). The mental health of Canadians with self-reported learning disabilities. Journal of Learning Disabilities, 42(1), 24-40.

[18] Winne, P. H., \& Hadwin, A. F. (1998). Studying as self-regulated learning. In D. J. Hacker, J. Dunlosky, \& A. C. Graesser (Eds.), Metacognition in educational theory and practice (pp. 277-304). Mahwah, NJ: Lawrence Erlbaum
Associates.

[19] Zimmerman, B. J. (2008). Investigating self-regulation and motivation: Historical background, methodological developments, and future prospects. American Educational Research Journal, 45, 166-183. 